\title{
Focus on spatial cognition
}

\author{
This month, Nature Neuroscience presents a special issue featuring reviews and opinion pieces on the theme of \\ spatial cognition.
}

$\mathbf{F}$ rom an evolutionary perspective, navigation - the ability to estimate one's own position and track and plan one's own path in physical space-is key to survival. It allows animals as diverse as ants, salmons, turtles and bats to locate food sources, find mates or avoid threats. In mammalian brains, the hippocampal-entorhinal region, well known for its role in explicit memory, has emerged as an essential node in the navigation system, contributing to the formation of a cognitive map of the outside world. In this month's special issue, Nature Neuroscience has put together a series of reviews and opinion pieces on the neural basis of spatial cognition. Together, these pieces suggest that the study of spatial cognition can be seen as a test bed for understanding some of the fundamental neural operations that underlie other forms of cognition.

All one needs to navigate is a map, a compass and a way to estimate distances traveled over a particular amount of time. There are cellular elements in the mammalian brain that assume these specific functions; they work together to build an internal map of space and keep track of one's position on it. The most well known of these elements are place, head direction and grid cells. In a Commentary on page 1448, Edvard and May-Britt Moser and Bruce McNaughton offer a history of the field of spatial navigation with a focus on path integration and share their thoughts on the outstanding questions that will be important to address in the future.

Cellular elements do not work independently of each other, and, like other cognitive processes, navigation relies on synaptic connections between cells, the integration of synaptic inputs and synaptic plasticity rules. In a Review on page 1483, Schmidt-Hieber and Nolan discuss the synaptic integrative mechanisms underlying spatial cognition.

Spatial cell types found in the hippocampal formation are defined by the tuning of their firing patterns with respect to certain features of physical space. On page 1474 of this issue, Giocomo, Ganguly and colleagues discuss how to best define physiological cells types. They recommend using more unbiased statistical methods than fitting predefined tuning curves to capture the mixed, heterogenous and dynamic selectivity of cells found in the medial entorhinal cortex, which, they argue, exists to implement a general-purpose learning machine that adapts though plasticity to current behavioral demands.

Head direction cells form the internal compass needed for efficient navigation. In a Perspective on page 1465, Cullen and Taube review our current understanding of the sense of direction in the real world and in virtual reality. They discuss how early vestibular systems encode head and body motions and integrate multimodal information for thalamus and cortex to then compute headings and generate estimates of self-motion.
Our ability to orient is anchored by the objects that surround us, which are used as landmarks on the cognitive map. Parallel visual pathways are thought to segregate spatial and object information into different streams that are fed to distinct temporal cortical regions before converging in the hippocampus to serve localization and memory processes. On page 1493, Knierim and Connor review the function of the ventral visual pathway and associated perirhinal and lateral entorhinal cortices. They argue that these object-related regions integrate precise object information and spatial information to encode the spatial relationships between objects, and between objects and self, that are important for navigation and spatial memory processes.

Are the many findings on spatial representation in animals conserved in humans? On page 1504, Epstein, Spiers and colleagues review spatial coding, landmark anchoring and route planning in humans and discuss how these functions essential to navigation may apply to nonspatial cognitive domains to enable navigation in nonphysical spaces.

A common thread that is woven throughout the pieces in this Focus is the involvement of the hippocampus in spatial cognition. A Viewpoint on page 1434 highlights the role of the hippocampus in memory, navigation and spatial cognition. Led by John Lisman, five researchers share their views on several key issues related to spatial information processing, its relationship to memory and time, and whether its mechanisms may serve to represent other dimensions of experience.

In this Focus introduction, we would also like to pay tribute to Howard Eichenbaum, who unexpectedly passed away recently (see Obituary, page 1432). Howard shaped the fields of memory and navigation in unique ways. He was an active scientist, prolific author and an exemplary referee, always providing constructive comments aimed at helping authors improve their work. He was versatile, weighing in on research studies on spatial and nonspatial cognitive processes, in animals or humans. Because of the veil of anonymity, good refereeing is rarely recognized. Not only was Howard a great neuroscientist but he helped countless researchers, without them even knowing, through his work as a referee. The journal and the field are indebted to his many contributions. Some of his last written words as a scientist can be read in this issue's Viewpoint article.

We of course hope that this series of articles will stimulate those working in the field, but because of its many ramifications, this series on spatial cognition should be of interest to many other neuroscientists. Thus, we are confident that many of the pieces presented in this issue will be useful and inspire many of our readers working outside the field of navigation. We also very much look forward to publishing articles on spatial cognition in the future that will push the boundaries of the field even further. 